Ann. Biol. anim. Bioch. Biophys., 1977, 17 (5 A), 655-659.

\title{
Cinétique des lipides chez le lapin hypertendu
}

\author{
par M. L. MICHAILOV
}

\author{
Instifut central de Recherches sur le Cœur \\ ef la Circulation sanguine, \\ Académie des Sciences de la R. D. A. \\ Berlin-Buch 1115 République Démocratique d'Allemagne.
}

Summary. Kinetics of lipids in the hypertensive rabbit.

A triglyceride emulsion $(0.5 \mathrm{~g} / \mathrm{kg})$ rich in linoleic and oleic acids was given by intravenous route to normal rabbits or to those made hypertensive by renal compression. The triglycerides behaved differently in the hypertensive than in normal subjects. In normal animals, linoleic and oleic acid titers in plasma triglycerides rose between 6 and $30 \mathrm{~min}$ after administration of the lipid emulsion. No increase in triglyceride fatty acid content was observed in hypertensive animals. The hypertension changed the kinetics of plasma triglycerides which were eliminated rapidly from the blood. This result shows the effect of different factors in experimental hypertension, and in particular the increased need of unsaturated fatty acids in the organism.

Dans des recherches antérieures (Michailov, 1974), nous avons déjà constaté des altérations dans le métabolisme lipidique dans les conditions d'hypertension. Les décalages dans la composition et la dynamique des métabolites lipidiques sont en relation avec les exigences énergétiques accrues, et dépendent de la durée et du degré d'hypertension. Nous étudions ici la cinétique de triglycérides injectés par voie intraveineuse sous forme d'émulsion, chez le lapin hypertendu ef chez l'animal normal.

\section{Matériel et méthodes.}

Les recherches ont été réalisées sur 20 lapins soumis à une hypertension expérimentale et 10 lapins témoins. Les animaux des deux sexes, de race indéterminée, proviennent d'un élevage appartenant à l'Institut. Ils sont âgés de 12 mois ef ont un poids moyen d'environ $4 \mathrm{~kg}$. Ils reçoivent une alimentation standard en cages individuelles.

L'hypertension rénale est produite par la méthode des capsules de cellophane (Page, 1939 ; Meier et Zbinden, 1946). Deux semaines après la pose de la capsule sur 
un des reins, on pratique l'ablation de l'autre rein. Deux semaines plus tard, une hypertension se manifeste.

L'expérience est réalisée après stabilisation de l'hypertension, avec pression à la systole de $158 \pm 5$ torr contre une valeur moyenne initiale d'environ $102 \pm 2$ torr.

Avant l'expérience, les animaux sont soumis à un jeûne nocturne de $12 \mathrm{~h}$.

On injecte rapidement aux animaux une émulsion de lipides $(0,5 \mathrm{~g} / \mathrm{kg})$ par voie intraveineuse. Cette émulsion contient : 20 p. 100 d'huile de soja, 1,5 p. 100 de phosphatide de soja ef 5 p. 100 de xylite. La composition en acides gras de l'huile de soja utilisée est la suivante, en p. 100 en poids : 53,6 d'acide linoléique, 21,7 d'acide oléique, 12,7 d'acide palmitique, 8 d'acide linolénique et 4 d'acide stéarique.

Les prises de sang ont lieu avant, puis 6,30 et $60 \mathrm{mn}$ après l'administration des lipides, à l'aide d'une sonde implantée quelques jours auparavant dans l'artère carotide. L'anticoagulant utilisé est le fluorure de sodium.

Les lipides du plasma sont extraits par un mélange de chloroforme-méthanol (2: 1), à raison de $20 \mathrm{ml}$ pour $1 \mathrm{ml}$ de plasma, puis séparés par chromatographie en couche mince sur gel de silice (Luis-Ferdinand ef al., 1967). Les acides gras des triglycérides sont séparés par chromatographie en phase gazeuse. Les conditions de la chromatographie en phase gazeuse sont les suivanies: colonne en verre de $2 \mathrm{~m} \times 2,7 \mathrm{~mm}$; phase stationnaire : 10 p. 100 diéthylène-glycol-succinate sur chromosorbe $W, 60$ à 80 mesh ; gaz porteur : azote $2,8 \mathrm{l} / \mathrm{h}$; température $190^{\circ} \mathrm{C}$ dans la colonne (isotherme); détecteur à ionisation à flamme.

\section{Résultats.}

La composition en acides gras des triglycérides plasmatiques des animaux témoins à jeûn est, comme le montre le tableau 1, influencée par l'injection de l'émulsion de lipides; on note une augmentation des teneurs en acides gras prépondérants dans l'émulsion administrée. En effet, la teneur en acide linoléique subit les changements les plus importants : +80 p. 100 à la $6^{e} \mathrm{mn},+62$ p. 100 à la $30 \mathrm{e} \mathrm{mn}$; par contre,

TABLEAU 1

Composition en acides gras des triglycérides plasmatiques (en p. 100 en poids) avant ef 6,30 ef $60 \mathrm{mn}$ après l'injection intraveineuse de lipides chez les animaux normotoniques. $\bar{x}=$ valeur moyenne : $S=$ déviation standard

\begin{tabular}{|c|c|c|c|c|c|c|c|c|}
\hline \multirow{2}{*}{ Acides gras } & \multicolumn{2}{|c|}{0} & \multicolumn{2}{|c|}{$6^{\mathrm{e}} \mathrm{mn}$} & \multicolumn{2}{|c|}{$30^{\mathrm{e}} \mathrm{mn}$} & \multicolumn{2}{|c|}{$60^{\mathrm{e}} \mathrm{mn}$} \\
\hline & $\overline{\mathbf{x}}$ & $S$ & $\overline{\mathbf{x}}$ & $\mathrm{S}$ & $\overline{\mathbf{x}}$ & $\mathrm{S}$ & $\overline{\mathbf{x}}$ & $\mathrm{S}$ \\
\hline $\begin{array}{l}\text { Linoléique } \ldots \ldots \ldots \ldots \ldots \\
\text { Oléique } \ldots \ldots \ldots \ldots \ldots \\
\text { Palmitique. } \ldots \ldots \ldots \ldots \ldots \\
\text { Linolénique } \ldots \ldots \ldots \ldots \ldots \\
\text { Stéarique } \ldots \ldots \ldots \ldots \ldots \ldots\end{array}$ & $\begin{array}{r}15,3 \\
25,2 \\
26,8 \\
3,4 \\
4,0\end{array}$ & $\begin{array}{l}1,7 \\
2,6 \\
2,5 \\
0,6 \\
0,8\end{array}$ & $\begin{array}{r}27,3^{*} \\
32,3^{*} \\
24,5 \\
3,5 \\
3,2\end{array}$ & $\begin{array}{l}2,5 \\
3,4 \\
2,8 \\
0,7 \\
0,5\end{array}$ & $\begin{array}{r}25,8^{*} \\
29,3^{*} \\
26,0 \\
4,4 \\
2,8\end{array}$ & $\begin{array}{l}2,5 \\
3,0 \\
2,8 \\
0,7 \\
0,6\end{array}$ & $\begin{array}{r}14,9 \\
25,6 \\
27,1 \\
5,7 \\
3,8\end{array}$ & $\begin{array}{l}1,6 \\
3,1 \\
2,6 \\
0,8 \\
0,7\end{array}$ \\
\hline
\end{tabular}

* différence significative. 
à la 60 e mn elle est revenue à sa valeur initiale (fig. 1). L'acide oléique, qui est l'acide gras le plus représenté dans l'émulsion après l'acide linoléique, subit des variations dans le même sens : +28 p. 100 à la 6 e $\mathrm{mn}$, +16 p. 100 à la $30 \mathrm{e} \mathrm{mn}$ et un retour à la valeur initiale à la $60^{\mathrm{e}} \mathrm{mn}$ (fig. 2). Les différences pour ces deux acides sont hautement significatives $(P<0,01)$. Les teneurs des autres acides gras constitutifs de triglycérides plasmatiques ne montrent aucune différence significative, quel que soit le temps considéré après l'injection.

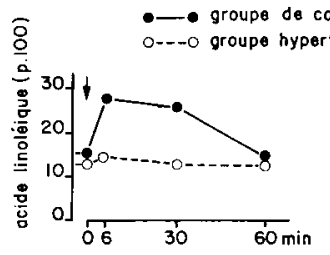

FIG. 1.

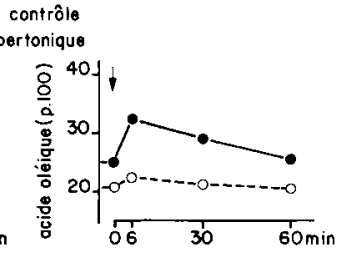

FIG. 2 .

FIG. 1. - Cinétique de la teneur en acide linoléique des triglycérides du plosma sanguin oprès l'injection intraveineuse de l'émulsion lipidique chez des animaux normotoniques ef hypertendus.

FIG. 2. - Cinétique de la teneur en acide oléique des triglycérides du plasma sanguin après l'injection intraveineuse de l'émulsion lipidique chez des animaux normotoniques et hypertendus.

Chez les animaux hypertendus nous n'avons constaté aucune variation cinétique des acides gras des triglycérides plasmatiques (tabl. 2). Les faibles modifications demeurent dans la limite des valeurs initiales (fig. 1 et 2).

\section{TABLEAU 2}

Composition en acides gras des triglycérides plasmatiques (en p. 100 en poids) avant ef 6,30 ef $60 \mathrm{mn}$ après l'injection intraveineuse de lipides chez les animaux présentont une hypertension expérimentale. $\bar{x}=$ valeur moyenne; $S=$ déviation standard.

\begin{tabular}{|c|c|c|c|c|c|c|c|c|}
\hline \multirow{2}{*}{ Acides gras } & \multicolumn{2}{|c|}{0} & \multicolumn{2}{|c|}{$6^{e} \mathrm{mn}$} & \multicolumn{2}{|c|}{$30^{\mathrm{e}} \mathrm{mn}$} & \multicolumn{2}{|c|}{$60^{\mathrm{e}} \mathrm{mn}$} \\
\hline & $\bar{x}$ & $\mathrm{~S}$ & $\overline{\mathbf{x}}$ & $\mathrm{S}$ & $\bar{x}$ & $\mathrm{~S}$ & $\overline{\mathbf{x}}$ & $\mathrm{S}$ \\
\hline $\begin{array}{l}\text { Linoléique } \ldots \ldots \ldots \ldots \ldots \\
\text { Oléique } \ldots \ldots \ldots \ldots \ldots \\
\text { Palmitique } \ldots \ldots \ldots \ldots \ldots \ldots \\
\text { Linolénique } \ldots \ldots \ldots \ldots \ldots \\
\text { Stéarique } \ldots \ldots \ldots \ldots \ldots \ldots\end{array}$ & $\begin{array}{r}12,7 \\
21,8 \\
32,2 \\
2,3 \\
9,8\end{array}$ & $\begin{array}{l}1,0 \\
2,2 \\
3,5 \\
1,0 \\
1,0\end{array}$ & $\begin{array}{r}14,2 \\
22,6 \\
32,1 \\
3,3 \\
9,6\end{array}$ & $\begin{array}{l}1,2 \\
2,5 \\
3,4 \\
0,8 \\
0,9\end{array}$ & $\begin{array}{r}13,0 \\
21,3 \\
32,4 \\
4,2 \\
9,3\end{array}$ & $\begin{array}{l}1,3 \\
2,5 \\
3,8 \\
0,8 \\
1,0\end{array}$ & $\begin{array}{r}12,8 \\
20,5 \\
33,7 \\
5,1 \\
9,5\end{array}$ & $\begin{array}{l}2,3 \\
2,8 \\
3,6 \\
1,0 \\
0,9\end{array}$ \\
\hline
\end{tabular}

\section{Discussion et conclusions.}

Nos résultats montrent que la captation des triglycérides exogènes plasmatiques par les tissus hépatiques et extrahépatiques dépend de l'état fonctionnel de l'orga- 
nisme et que des conditions d'hypertension expérimentale la modifient. La captation des triglycérides par les tissus exige qu'ils soient hydrolysés par la lipoprotéine-lipase. On sait, depuis longtemps, grâce à des recherches faites avec des acides gras marqués (Nestel, Bezman ef Havel, 1962) que les divers acides gras des triglycérides disparaissent de la circulation sanguine avec des vitesses différentes ; par exemple, l'acide linoléique est capté par les tissus plus rapidement que l'acide palmitique. Des résultats plus récents indiquent que chez l'animal au repos l'acide oléique est oxydé plus rapidement que l'acide stéarique ; par contre, au cours de l'exercice, ce dernier acide est métabolisé de façon plus intensive (Hagenfeldł et Wahren, 1975).

La diminution de la concentration de triglycérides dans le plasma lors d'une activité physique accrue est liée à une augmentation de la consommation d'acides gras non saturés.

L'activité physique est un facteur essentiel pour la régulation du métabolisme lipidique. L'augmentation de la concentration des acides gras libres dans le plasma sanguin après un apport exogène de triglycérides chez l'hypertendu est plus importante qu'à l'état normal ; cette constatation laisse supposer l'importance de ces métabolites en tant que fournisseurs d'énergie (Michailov et al., 1971).

Nos résultats semblent indiquer une utilisation accrue des acides gras non saturés, tout particulièrement des acides gras essentiels. On pourrait comparer l'hypertension à un état de besoin permanent d'acides gras polyinsaturés par suite de leur importance fonctionnelle ou bien de leur oxydation accrue, mais des expériences complémentaires devraient confirmer ce point. On doit expliquer le comportement des triglycérides dans le plasma sanguin par les diverses exigences fonctionnelles posées par les conditions d'hypertension, par exemple une activité accrue du foie ou des systèmes enzymatiques. Nos recherches rendent évidentes les différences qui existent dans la cinétique des triglycérides chez des animaux hypertendus ef chez des animaux normotoniques. Les décalages ont une certaine relation avec le degré et la durée de l'hypertension, ainsi qu'avec le développement de leurs mécanismes adaptatifs. On peut admettre qu'ils contribuent à caractériser les paramètres du mécanisme d'hypertension.

Reçu en octobre 1976. Accepté en février 1977.

\section{Références}

HAGENFELDT L., WAHREN J., 1975. Turnover of plasma free stearic and oleic acids in resting and exercising human subjects. Metabolism, 24, 1299-1304.

LUIS-FERDINAND R. T., THERRAULT P. G., BLATT W. F., MAGER M., 1967. Application of thinlayer chromatography to the quantitation of plasma neutral lipids and free fatty acids. Clin. chim. Acfa, 13, 773-787.

MEIER R., ZBINDEN P., 1946. Neues Verfahren zur experimentellen Erzeugung eines permanenten und reversiblen Hochdruck durch standardisierte Kompression der Niesre. Experientia, 2, 259-268.

MICHAILOV M. L., 1974. Modifications de la composition en acides gras libres du plasma de rat soumis à différents modes d'hypertension. Biomed. Express, 20, 393-397. 
MICHAILOV M. L., NITSCHKOFF S., HOLLSTEIN E., BAUMANN R., 1971. Über Veränderungen des Spektrums der veresterten Fettsäuren (Triglyzeride) im Blułplasma bei experimenteller nephrogener Hypertonie and der Ratte. Disch. Gesundh Wes., 26, 763-767.

NESTEL P. J., BEZMAN A., HAVEL P. J., 1962. Metabolism of palmitate and linoleate in intact dogs. Amer. J. Physiol., 203, 914-918.

PAGE I. H., 1939. A method for producing persistent hypertension by cellophane. Science, 89, 273 274. 